\title{
The Capability Index when Some Assumptions are not Satisfied: Analysis and Empirical Comparisons
}

\author{
PABlo José Moya Fernández a , JUAN Francisco Muñoz Rosas a , \\ ENCARNACIÓN ÁLVAREZ VERDEJO ${ }^{a}$ \\ a Universidad de Granada, Fctad. CC.EE., Campus de la Cartuja, s/n, 18071 Granada, España. E- \\ mail: pjmoyafernandez@ugr.es,jfmunoz@ugr.es, encarniav@ugr.es
}

\begin{abstract}
The process capability index (PCI) evaluates the ability of a process to produce items with certain quality requirements. The PCI depends on the process standard deviation, which is usually unknown and estimated by using the sample standard deviation. The construction of confidence intervals for the PCI is also an important topic. The usual estimator of the PCI and its corresponding confidence interval are based on various assumptions, such as normality, the fact that the process is under control, or samples selected from infinite populations. The main aim of this paper is to investigate the empirical properties of estimators of the PCI, and analyze numerically the effect on confidence intervals when such assumptions are not satisfied, since these situations may arise in practice.

Keywords: Proportion of Non-Conforming Items, Capability Analysis, Monte Carlo Simulation, Standard Deviation, Sample Range.
\end{abstract}

\section{El índice de capacidad cuando no se cumplen algunas hipótesis de partida: Análisis y comparaciones empíricas.}

\section{RESUMEN}

El índice de capacidad (PCI) evalúa la habilidad de un proceso para producir artículos con determinados requerimientos de calidad. El PCI depende de la desviación típica del proceso, la cual suele ser desconocida y estimada a partir de la desviación típica muestral. La construcción de intervalos de confianza para el PCI también es un tema relevante. El estimador estándar del PCI y su correspondiente intervalo de confianza están basados en varias hipótesis de partida, tal como normalidad, el hecho de que el proceso se encuentra bajo control, o muestras seleccionadas de poblaciones infinitas. El principal objetivo de este trabajo es investigar las propiedades empíricas de dos estimadores del PCI, y analizar numéricamente el efecto en los intervalos de confianza cuando no se cumplen tales hipótesis, puesto que estas situaciones pueden presentarse en la práctica.

Palabras clave: Proporción de artículos disconformes, Análisis de Capacidad, Simulación Monte Carlo, desviación típica, rango muestral.

Clasificación JEL: C13, C15, C16, L23

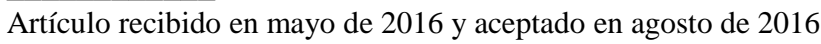

Artículo disponible en versión electrónica en la página www.revista-eea.net, ref. ə-34307 


\section{INTRODUCTION}

The wisdom is a key aspect within a production process of many companies. For instance, it allows evaluating: (i) the quality of items they produce; (ii) the number of items that meet certain conditions, etc. The interest on this issue is mainly motivated because the company aims to offer a product of quality to consumers, and which is able to build customer loyalty. Second, this interest is also justified by the fact that items must satisfy some quality specifications, which are generally imposed by external agents. For example, a regulation may require products with certain characteristics.

The aim of the Statistical Quality Control (SQC) is the evaluation and monitoring of quality of products (Chakraborti et al., 2008, Mitra 2008, Montgomery 2009). The main methodology of SQC is to select a quality characteristic of a product under study, and then take a sample of them to measure this quality characteristic for the items selected in the sample. The sample data are transformed into indicators, which are used to compare the quality of the selected products to fixed in advance quality specifications. This translates into a very valuable knowledge for the next phase, which is to make a decision to run the production process more efficiently. A first option is to take no action on the production process, in the case the evidences indicate that the process is under control. On the other hand, the knowledge based on the sample information may indicate that many items do not satisfy the quality requirements, and the instruction is to perform corrective actions to improve the production process. This is an example of how the sample data are transformed into information, which is used to have a better knowledge of the production process. Accordingly, this knowledge is transformed into wisdom, which in turn is used to make the production process works more effectively, and consequently, reduce costs and make the company more profitable.

The SQC is based on many quantitative techniques, such as control charts, acceptance sampling, process capability analysis, etc. This article focuses on the process capability analysis, which aims to evaluate the ability of a production process for the manufacture of acceptable products, i.e., products that meet the quality requirements. Such quality requirements are usually defined by the specification limits. An upper specification limit (USL) is the largest allowable value for the quality characteristic. Similarly, the lower specification limits (LSL) is the smallest allowable value for the quality characteristic. It is quite common that the quality requirements are based on both lower and upper specification limits. Specification limits are also known as tolerances, i.e., they indicate ranges of acceptance of a given item.

A process capability analysis can be based on many techniques (control charts, histograms, probability plotting, etc), but the most common indicator is the process capability index (PCI), also named as the process capability ratio. 
The PCI can be of a great importance for many companies, and for this reason it has been extensively studied (Kotz and Lovelace 1998, Kotz and Johnson 2002, Spiring et al., 2003, Montgomery 2009 p.351, etc). For instance, the PCI may determine whether the process is valid in the production of an item under certain quality requirements, and an early diagnosis is an important step to reduce costs and improve market. Therefore, the PCI evaluates the ability of the process to produce items within the specification limits, i.e., the ability to produce acceptable items. Note that PCI only holds if the corresponding process is in a state of statistical control, i.e., the process is "in control" (see Montgomery 2009, p.181, Evans and Lindsay 1999, p.321, Jensen et al., 2006). See also the Assumption A3 in Section 2.

The process capability is directly related to the process variability. For instance, the PCI compares the specification limits with the natural variability of a process (Besterfield 2014), which is based on the process standard deviation (denoted as $\sigma$ in this paper). It is quite common to consider a width of the process natural variability equal to $6 \sigma$ (Chakraborti el al. 2008, Mitra 2008, Montgomery 2009 , etc). An argument in favour of the $6 \sigma$ criterion is the fact that the "sixsigma" techniques are topics of increased interest in many companies, hence the "six-sigma" is recently receiving a relevant expectation (Breyfogle 2003). Finally, note that control charts (Chen 1997, Montgomery 2009 p.184, Muñoz et al., 2016) and other many methods in SQC are based on this criterion.

In practice, the parameter $\sigma$ is often unknown. The usual solution is to select a sample when we are confident that the process is in control (see the Assumption A3 in Section 2), and then the information collected from the sample is used to estimate $\sigma$. Many methods have been proposed for this purpose, but the best known and used of them are based on the sample standard deviation and the sample range (Chakraborti 2008, Chen 1997, Duncan 1986, Jones et al., 2001, Luko 1996, Montgomery 2009 pp. 229 and 253, Ott 1975, Vanderman 1999, Wheeler 1995, Woodall and Montgomery 2000, Muñoz et al., 2016). We consider the estimator based on the sample standard deviation, since the traditional confidence intervals for the PCI are based on this estimator.

The PCI depends on the process standard deviation, and for this reason, the PCI is usually unknown in practice and its estimation is required. For instance, Álvarez et al. (2015) analyze the effect on the PCI of different estimators based on multiples samples ( $m$ samples), which is the situation when working with control charts and other many techniques in SQC. However, many other situations may arise in practice, and for this reason they are analyzed in this paper. First, this paper assumes a single sample. Note that the expressions of estimators are different under this situation, which may arise when we are interested on analyzing the PCI at a given time. A second contribution with respect to Álvarez et al. (2015) is the fact that confidence intervals for the PCI (Kotz and Johnson 
1993, p.43) are investigated. Third, we analyze the effect on the estimation of the PCI and the corresponding confidence intervals when different estimators of the process standard deviation are considered. In particular, we consider the biased and the unbiased estimators of the process standard deviations based on the sample standard deviation. Fourth, the main aim of this paper is to analyze the PCI when some assumptions are not entirely satisfied. In particular, the estimator of the PCI and/or its corresponding confidence interval assumes that: (i) the data follow a Normal distribution; (ii) samples are extracted from an infinite population; and (iii) the process is under control. These assumptions are described in Section 2. Note that the failure of these assumptions is an option in practice. For instance, the associated distributions are statistically valid when the variables are independent and identically distributed. However, situations may arise where this assumption is not satisfied. For instance, the production of many companies is stored in finite lots, from which samples are extracted for the application of different SQC techniques. The "acceptance sampling" is an example where samples are selected from finite populations (Montgomery 2009 p.629, Aslam et al., 2015, Rao et al., 2016, Nezhad and Niaki 2013, etc.) If samples are selected from finite populations, the usual confidence intervals for the PCI, which are based upon infinite populations, could have a poor performance. A contribution of this paper is to analyze numerically the usual confidence intervals for the PCI, which assume infinite populations. However, we consider a common situation in practice (finite populations) in order to investigate the effect of the lack of this assumption. Finally, we also compare results derived from center and off-center processes. A detailed description of the contributions of this paper can be seen in Sections 3.1 and 3.3.

This paper is organized as follows. In Section 2, we first define the PCI, and different estimators based on the sample standard deviation are also introduced. The construction of confidence intervals is also addressed in this section. The most common assumptions of estimators and confidence intervals for the PCI are also described. Note that the different methods are based on a single sample. In section 3 , we analyze numerically the effect on estimators and confidence intervals when the described assumptions are not entirely satisfied. The proposed empirical studies are carried out by using Monte Carlo simulation. Artificial data sets with different properties are considered, besides a real data set commonly used in the context of SQC. Our findings are also summarized in Section 4 .

\section{ESTIMATION OF THE PROCESS CAPABILITY INDEX}

\subsection{Definition}

Let $x$ be a quality characteristic associated to a process that operates with a mean $\mu$ and a standard deviation $\sigma$. The PCI is defined as the ratio of the width of 
the specification limits and the width of natural tolerance limits of the process, i.e.

$$
C_{p}=\frac{U S L-L S L}{6 \sigma},
$$

where USL and LSL are, respectively, the upper and the lower specification limits, and $6 \sigma$ represents the natural variability of the process. We observe that $C_{p}$ compares the natural variability of the process with the permissible range of values in the process, which is defined by the specification limits.

The PCI describes how well the process produces acceptable items, i.e., the proportion of items that meet the specification limits. The process will be classified as unfit if the PCI is less than 1, since the width of the specification limits is smaller than the natural variability of the process, and this implies that the process produces an excessive proportion of non-conforming items. Corrective actions on the process are required in this situation. For example, if there is a margin in the quality requirements, the easier corrective action to reduce the proportion of non-conforming items is to increase the range of the specification limits. Otherwise, some improvements must be introduced in the process to reduce the process variability and the proportion of non-conforming items.

The process is suitable to produce items within the specification limits if the PCI is larger than 1 . The proportion of non-conforming items should be small in this situation, and it decreases as the value of the PCI increases. In addition, any change of the parameters associated to the process will have a smaller impact on the proportion of non-conforming items.

The process is marginally suitable to produce items within specification limits if the PCI equal to 1 (Kotz and Johnson 1993, Chakraborti 2008 and Chen 1997). This situation is very sensitive to any change in the parameters associated to the process, since this issue can produce a significant increase in the proportion of non-conforming items.

From equation (1) we observe that $C_{p}$ uses bilateral specification limits, which is the most common situation. However, one-sided capability indexes can be easily defined (Montgomery 2009, p.352). For simplicity, we consider bilateral specification limits in this paper, but the extension of this study to the case of one-sided limits is quite straightforward, and similar results are also expected.

The PCI defined by expression (1) assumes that the midpoint of the specification limits is equal to the process mean $\mu$. If this is the situation, it is said that the process is centered. For off-center processes, the PCI should take into account the position of the process mean relative to the specification limits. For this reason, the process capability index for an off-center process $\left(\mathrm{PCI}_{k}\right)$ is defined as 


$$
C_{p k}=(1-k) C_{p}
$$

where

$$
k=\frac{\left|\mu_{0}-\mu\right|}{(U S L-L S L) / 2},
$$

and $\mu_{0}=(L S L+U S L) / 2$ is the midpoint of the specification limits. The value $k=0$ indicates that the production process is centered, i.e., $\mu_{0}=\mu$ and $C_{p k}=C_{p}$. If $0<k<1$, the distance between $\mu_{0}$ and $\mu$ is less than half the interval width defined by the specification limits, and $C_{p k}<C_{p}$. If $k \geq 1$, the distance between $\mu_{0}$ and $\mu$ is greater than half the interval width defined by the specification limits, and this implies a large proportion of non-conforming items.

\subsection{Common assumptions}

We assume a single sample with size $n$, where $x_{1}, \ldots, x_{n}$ denote the sample values of the quality characteristic $x$. The sample mean is defined as

$$
\bar{x}=\frac{1}{n} \sum_{i=1}^{n} x_{i},
$$

whereas the sample standard deviation is defined as

$$
S=\left(\frac{1}{n-1} \sum_{i=1}^{n}\left(x_{i}-\bar{x}\right)^{2}\right)^{1 / 2} .
$$

The estimation of the PCI and/or the corresponding confidence intervals are generally based on the following assumptions:

(A1). The quality characteristic $x$ follows a Normal distribution, i.e., $x \sim N(\mu, \sigma)$.

(A2). The variables associated to the sample values $x_{1}, \ldots, x_{n}$ are independent and identically distributed. For instance, this assumption holds if the sample is selected from an infinite population.

(A3). The process is "in control" (see Montgomery 2009, p.181, Evans and Lindsay 1999, p.321, Jensen et al., 2006). Assuming A1 and A2, this implies that both parameters of the Normal distribution ( $\mu$ and $\sigma$ ) are under control, i.e., the statistic $S$ is within the control limits

$$
\begin{aligned}
U C L_{\sigma} & =\left(c_{4}[n]+\frac{c}{\sqrt{2(n-1)}}\right) \sigma \\
L C L_{\sigma} & =\left(c_{4}[n]-\frac{c}{\sqrt{2(n-1)}}\right) \sigma
\end{aligned}
$$


where $L C L_{\sigma}$ denotes the lower control limit, $U C L_{\sigma}$ is the upper control limit, and $c_{4}[n]$ is a function that depends on the sample size (see Chen 1997). Some values of $c_{4}[n]$ for various sample sizes have been tabulated in Appendix VI of Montgomery (2009). The value $c$ is a constant used to define the distance of the control limits from the center line, which is fixed at $C L_{\sigma}=c_{4}[n] \sigma$ in this situation. Note that it is quite common to consider the value $c=3$ (see Montgomery 2009, p.181, Evans and Lindsay 1999, p.321, Jensen et al., 2006). Similarly, the parameter $\mu$ is in control if the statistic $\bar{x}$ is within the control limits

$$
\begin{aligned}
U C L_{\mu} & =\mu+c \frac{\sigma}{\sqrt{n}}, \\
L C L_{\mu} & =\mu-c \frac{\sigma}{\sqrt{n}},
\end{aligned}
$$

where the value $c=3$ is usually considered.

\subsection{Estimation of the process capability index}

The process standard deviation $\sigma$ is usually unknown, hence that the PCI defined by equation (1) is also unknown. The customary estimator for $C_{p}$ is obtained by substituting $\sigma$ by its estimator into (1). We consider estimators based on the sample standard deviation, since the most common confidence intervals for the PCI are based on this estimator. The usual estimator of $\sigma$ is defined by:

$$
\hat{\sigma}=S
$$

It is well known that $\hat{\sigma}$ is not unbiased. For this reason, and assuming A2, it is common to use the estimator

$$
\hat{\sigma}_{c 4}=\frac{S}{c_{4}[n]}
$$

when the sample sizes are small. Note that $c_{4}[n]$ is used because it provides an unbiased estimator for the process standard deviation $\sigma$ (Duncan 1986, Luko 1996, Wheeler 1995, and Woodall and Montgomery 2000).

Following equation (1), the estimators of $C_{p}$ based on the previous estimators of $\sigma$ are defined as:

$$
\begin{aligned}
& \hat{C}_{p}=\frac{L S E-L I E}{6 \hat{\sigma}} \\
& \hat{C}_{p . c 4}=\frac{L S E-L I E}{6 \hat{\sigma}_{c 4}}
\end{aligned}
$$


For off-center processes, the estimators of $C_{p k}$ are given by

$$
\hat{C}_{p k}=(1-\hat{k}) \hat{C}_{p}
$$

and

$$
\hat{C}_{p k . c 4}=(1-\hat{k}) \hat{C}_{p . c 4}
$$

where

$$
\hat{k}=\frac{\left|\mu_{0}-\hat{\mu}\right|}{(U S L-L S L) / 2},
$$

and $\hat{\mu}=\bar{x}$ is the sample mean.

From expressions (4) and (5) we observe that the precision of the estimation of the PCI is directly related to the precision of the estimation of the process standard deviation. In this paper, we investigate the empirical performance of the estimators $\hat{\sigma}$ and $\hat{\sigma}_{c 4}$, and such results are compared to the empirical performance of the estimators of PCI $\left(\hat{C}_{p}\right.$ and $\left.\hat{C}_{p . c 4}\right)$ and $\mathrm{PCI}_{\mathrm{k}}\left(\hat{C}_{p k}\right.$ and $\left.\hat{C}_{p k . c 4}\right)$.

\subsection{Estimation using confidence intervals}

The construction of confidence intervals for $\mathrm{PCI}$ and $\mathrm{PCI}_{\mathrm{k}}$ has been extensively discussed (Kane 1986, Heavlin 1988, Chou et al., 1990, Chou and Owen 1989, Li et al., 1990). However, such references assume A1, A2, and A3. In particular, we know that

$$
\frac{(n-1) \hat{\sigma}^{2}}{\sigma^{2}} \rightarrow \chi_{n-1}^{2}
$$

where $\hat{\sigma}^{2}=S^{2}$. If we consider the estimator $\hat{C}_{p}$ defined by (4), and use the distribution given by (6), it can be easily seen that a $(1-\alpha) \%$ confidence interval for PCI is defined by (Kotz and Johnson 1993):

$$
[L, U]=\left[\frac{\chi_{n-1, \alpha / 2}}{\sqrt{n-1}} \hat{C}_{p}, \frac{\chi_{n-1,1-\alpha / 2}}{\sqrt{n-1}} \hat{C}_{p}\right],
$$

where $\chi_{n-1, a}$ is the square root of the $a$ th quantile of the chi-square distribution. In this paper, we analyze the confidence interval for the PCI based on the estimator $\hat{C}_{p . c 4},\left[L_{c 4}, U_{c 4}\right]$, which is similarly defined, i.e., we have to substitute $\hat{C}_{p}$ by $\hat{C}_{p . c 4}$ into (7). The confidence interval for $C_{p k}$ suggested by Heavlin (1988) is given by $\left[L_{k}, U_{k}\right]$, where

$$
L_{k}=\hat{C}_{p k}-Z_{1-\frac{\alpha}{2}}\left(\frac{n-1}{9 n(n-3)}+\hat{C}_{p k}^{2}\left(1+\frac{6}{n-1}\right)\right)^{\frac{1}{2}}
$$


and

$$
U_{k}=\hat{C}_{p k}+Z_{1-\frac{\alpha}{2}}\left(\frac{n-1}{9 n(n-3)}+\hat{C}_{p k}^{2}\left(1+\frac{6}{n-1}\right)\right)^{\frac{1}{2}},
$$

where $Z_{a}$ is the $a$ th quantile of the standard normal distribution. The confidence interval for $C_{p k}$ based on $\hat{\sigma}_{c 4}$, $\left[L_{k . c 4}, U_{k . c 4}\right]$, is similarly defined. The confidence intervals for $C_{p k}$ are also analyzed by Chou et al. (1990), Kushler and Hurley (1991), Franklin and Wasserman (1992), Guirguis and Rodriguez (1992), Dovich (1992), Nagata and Nagahata (1994), etc.

The aforementioned confidence intervals are based on the Assumptions A1, A2, and A3. However, situations may arise where such assumptions are not entirely satisfied. For instance, the "acceptance sampling” theory in SQC assumes the existence of finite lots, i.e., A2 is not satisfied in this situation. We could be interested on a capability analysis, and the sample data for this study could have been taken from such finite lots. If the size of the finite lots is large in comparison to the sample size, the confidence intervals based upon infinite populations can be used, since the use of the approximation to an infinite population is justified. However, this required assumption is not entirely satisfied otherwise. The effect of this approximation for different situations has not been investigated. In addition, the assumptions A1 and A3 could fail in practice. In Section 3, we analyze numerically the effect on the estimators and confidence intervals for the capability index when the assumptions A1, A2 and A3 are not entirely satisfied. For this purpose, we consider scenarios with different characteristics, and use Monte Carlo simulation.

\section{MONTE CARLO SIMULATION STUDIES}

Estimators and confidence intervals for PCI and $\mathrm{PCI}_{\mathrm{k}}$ are described in this paper. They can be based on different estimators of the process standard deviation, hence they may have a different performance under certain situations, such as samples with small sizes. First, this issue is investigated in this section. In addition, the usual confidence intervals for PCI and $\mathrm{PCI}_{\mathrm{k}}$ are based on the Assumptions A1, A2, and A3, but such assumptions may not appear in practice. We also analyze numerically the effect of the lack of the commented assumptions. Finally, we also compare the performance of estimators and confidence intervals based on both center and off-center processes.

\subsection{Description of data and scenarios used in simulation studies}

Three different scenarios have been considered. First, the sample data are randomly selected from a probabilistic distribution for each simulation run. This implies that variables are independent and identically distributed, i.e., samples 
are selected from infinite populations (the Assumption A2 holds). The second scenario consists on samples selected from finite populations and using simple random sampling without replacement. In this case, $N$ values are randomly selected from the corresponding probabilistic distribution, and they are considered a "lot" (a finite population), from which samples are selected. Large sampling fractions $(f=n / N)$ are considered in order to set more obvious differences between the cases of finite and infinite populations. Small sampling fractions are also considered in order to analyze numerically the approximation from finite population to infinite population. The sizes of finite populations are given by $N=\{500,10000\}$. For infinite populations, the sample sizes $(n)$ range from 10 to 500, and the same values are considered when $N=10000$, which implies that the sampling fractions go from $0.1 \%$ to $5 \%$ in this situation. Finally, the values of $n$ range from 5 to 250 when $N=500$, which give sampling fractions with values between $1 \%$ and $50 \%$. Finally (the third scenario), the real data set "pistonrings" is also considered. This real data set is taken from the package "qcc" (Scrucca 2004) included in the statistical software R (see also Montgomery 2009). Pistonrings data contain values related to the inside diameter of piston rings for an automotive engine, and which are produced by a forging process. Following Harms and Duchesne (2006), we considered this real data set as a finite population (with $N=125$ ), from which samples are selected. In this case, we considered sampling fractions with values between $5 \%$ and $50 \%$.

The commented random values are generated from three different probabilistic distributions: Normal, Gamma and Uniform. The aim is to analyze the effect of the lack of Assumption A1. Random values from the Normal distribution are obtained with a mean $\mu=10$, and a standard deviation $\sigma=1$, and which represent, respectively, the mean and standard deviation of a quality characteristic. For the Gamma distributions, we considered the parameters $\alpha=100$ and $\theta=1 / 10$ of this distribution. Finally, random values from the Uniform distribution are selected between 8.26795 and 11.73205 . It can be easily seen that the selected distributions have the same mean $(\mu=10)$ and the same standard deviation $(\sigma=1)$. Various hypothesis tests indicate that the pistonrings data follow a Normal distribution. In this situation the process mean is $\mu=74.00288$ and the standard deviation is $\sigma=0.01291$.

We can observe that the previous scenarios compare situations where the Assumption A1 and A2 hold to situations where such assumptions are not entirely satisfied. We also follow this idea for the Assumption A3. For this purpose, we first calculate the various estimators without checking if the process is under control, i.e., we considered all selected samples (A3 is not entirely satisfied in this situation). In addition, we also calculate the various estimators when the process is under control, i.e., we only consider the samples 
whose statistics $S$ and $\bar{x}$ are within the control limits (2) and (3), respectively. Such control limits are obtained using the constants $c=\{2.5,3\}$. Note that the percentage of samples under control is quite large, since data are extracted from the corresponding probabilistic distribution. This should be the situation in practice, i.e., most samples of a production process should be in control. For example, similar percentages are obtained for the real data set (pistonrings).

The specification limits (LSL and USL) are chosen such that the capability indices take the values $C_{p}=C_{p k}=1$, i.e., we considered center and off-center processes, and where $k$ takes the values $k=\{0,0.25,0.5\}$. In other words, for the generated populations we considered $L S L=7$ and $U S L=13$ when $k=0$ $\left(C_{p}=1 ; \mu_{0}=10\right), L S L=7$ and $U S L=15$ when $k=0.25\left(C_{p k}=1 ; \mu_{0}=\right.$ $11)$, and $L S L=7$ and $U S L=19$ when $k=0.5\left(C_{p k}=1 ; \mu_{0}=13\right)$. For the real data set (pistonrings), we considered the specification limits $L S L=$ 73.96414 and $U S L=74.04161$, since they also provide a capability index $C_{p}=1$ when $k=0$.

\subsection{Empirical measures used in simulation studies}

The various estimators and confidence intervals are analyzed in terms of different empirical measures, and which are obtained after $B=10000$ simulation runs. In particular, estimators are evaluated using the relative bias (RB) and the relative root mean square error (RRMSE). Note that they are the most common measures used to compare the accuracy of estimators (Rao et al., 1990, Silva and Skinner 1995). For instance, if we consider the estimator $\hat{C}_{p k}$ of the process capability index $C_{p k}$, the corresponding values of RB and RRMSE are defined by:

$$
R B_{P C I}=\frac{E\left[\hat{C}_{p k}\right]-C_{p k}}{C_{p k}}
$$

and

$$
\operatorname{RRMSE}_{P C I}=\frac{\sqrt{M S E\left[\hat{C}_{p k}\right]}}{C_{p k}},
$$

where

$$
E\left[\hat{C}_{p k}\right]=\frac{1}{B} \sum_{b=1}^{B} \hat{C}_{p k}(b)
$$

is the empirical expected value of $\hat{C}_{p k}$ based on the $B$ simulation runs, $\hat{C}_{p k}(b)$ denotes the value of $\hat{C}_{p k}$ at the $b$ th simulation run, and 


$$
\operatorname{MSE}\left[\hat{C}_{p k}\right]=\frac{1}{B} \sum_{b=1}^{B}\left(\hat{C}_{p k}(b)-C_{p k}\right)^{2}
$$

is the empirical mean square error of $\hat{C}_{p k}$. The values of RB and RRMSE defined by expressions (8) and (9) are similarly defined when estimating the standard deviation $\sigma$, and they are denoted as $R B_{S D}$ and $R R M S E_{S D}$.

Confidence intervals are evaluated using the empirical coverage rates of them and a $95 \%$ for the confidence level. For instance, if $L$ and $U$ are the lower and upper limits of the confidence interval for $C_{p k}$, the corresponding empirical coverage is defined by

$$
\text { Coverage }_{P C I}=\frac{1}{B} \sum_{b=1}^{B} I_{[L, U]}(b),
$$

where $I_{[L, U]}(b)$ denotes the value of a indicator variable at the $b$ th simulation run, and which takes the value 1 if $C_{p k}$ is within the interval $[L, U]$, and it takes the value 0 otherwise. Note that it is desirable that the empirical coverage rates take values close to the nominal confidence level (95\%). Estimators and confidence intervals are obtained using $\hat{\sigma}$ and $\hat{\sigma}_{c 4}$.

\subsection{Results derived from simulation studies}

First, estimators $\hat{\sigma}$ and $\hat{\sigma}_{c 4}$ are compared in Table 1 . It is expected to observe differences between them when the sample sizes are small.

Figures 1, 2 and 3 consider data selected from the Normal distribution, and the problem is to estimate $C_{p k}=1$, with $k=\{0,0.25,0.5\}$, respectively. The population size of finite populations in these figures is $N=500$. In addition, Figures 1, 2 and 3 include empirical measures when all samples are under control, and using the constant $c=2.5$ for the control limits. Second, the effect of different values of $k$ on the estimation of the capability index can be observed through Figures 1, 2 and 3.

Third, note that each plot contains results when: (i) all samples are considered; (ii) only samples under control are considered. This issue will allow us to analyze the effect on estimators and confidence intervals when the Assumption A3 is not entirely satisfied. Note that the case $c=3$ in Figures 1, 2, and 3 is omitted because the differences between the scenarios (i) and (ii) is small under this situation.

In addition, each plot of Figures 1, 2 and 3 contains both cases of finite $(N=500)$ and infinite populations, and which can be used to analyze the Assumption A2. This is the fourth aim in this study. Similarly (fifth study), we also consider the case of a finite population with size $N=10000$ and small sample sizes (Figure 4). In this situation, the approximation to an infinite 
population holds well, and comparisons to the previous scenarios can be made. Alternative parameters in Figure 4 remain the same as used in Figure 1: $C_{p k}=1$, with $k=0$, and $c=2.5$ for the control limits.

Table 1

Values of Relative Bias (RB (\%)) and Relative Root Mean Square Error (RRMSE(\%)) for estimators of $\sigma=1(\mathrm{SD})$ and $\mathrm{C}_{\mathrm{p}}=1(\mathrm{PCl})$, and empirical coverages (\%) of confidence intervals for $\mathrm{C}_{\mathrm{p}}=1(\mathrm{PCl})$. Sample data are selected from a Normal distribution with $\mu=10$ and $\sigma=1$ (infinite population). For the finite population, $\mathrm{N}=500$ data are selected from this distribution and then samples without replacement are selected from this data set.

\begin{tabular}{|c|c|c|c|c|c|c|c|c|c|c|c|}
\hline \multirow[b]{2}{*}{ Population } & \multirow[b]{2}{*}{$n$} & \multicolumn{2}{|c|}{$R B_{S D}$} & \multicolumn{2}{|c|}{$R R M S E_{S D}$} & \multicolumn{2}{|c|}{$R B_{P C I}$} & \multicolumn{2}{|c|}{$R_{R M S E_{P C I}}$} & \multicolumn{2}{|c|}{ Coverage $_{P C I}$} \\
\hline & & $\widehat{\sigma}$ & $\widehat{\sigma}_{c 4}$ & $\widehat{\sigma}$ & $\widehat{\sigma}_{c 4}$ & $\widehat{\boldsymbol{C}}_{p}$ & $\widehat{\boldsymbol{C}}_{p . c 4}$ & $\widehat{C}_{p}$ & $\widehat{C}_{p . c 4}$ & $\widehat{\boldsymbol{C}}_{p}$ & $\widehat{C}_{p . c 4}$ \\
\hline \multirow{6}{*}{ Infinite } & 5 & -6.4 & -0.8 & 34.0 & 35.1 & 25.2 & 18.0 & 67.3 & 61.3 & 95.5 & 94.3 \\
\hline & 10 & -2.8 & -0.1 & 23.0 & 23.3 & 9.3 & 6.4 & 30.9 & 29.3 & 95.3 & 94.8 \\
\hline & 15 & -1.8 & -0.1 & 18.4 & 18.6 & 5.7 & 3.9 & 22.1 & 21.4 & 95.4 & 95.1 \\
\hline & 20 & -1.3 & 0.0 & 16.0 & 16.1 & 4.1 & 2.8 & 18.3 & 17.8 & 95.3 & 95.0 \\
\hline & 25 & -0.9 & -0.1 & 14.2 & 14.3 & 3.1 & 2.1 & 15.7 & 15.4 & 95.4 & 95.0 \\
\hline & 50 & -0.5 & 0.0 & 10.1 & 10.1 & 1.5 & 1.0 & 10.6 & 10.5 & 95.0 & 94.9 \\
\hline \multirow{6}{*}{$\begin{array}{c}\text { Finite } \\
(N=500)\end{array}$} & 5 & -10.4 & -5.6 & 35.4 & 35.5 & 32.5 & 25.1 & 75.3 & 68.4 & 94.8 & 94.6 \\
\hline & 10 & -4.9 & -2.4 & 24.1 & 24.2 & 12.5 & 9.5 & 34.0 & 32.1 & 94.0 & 93.9 \\
\hline & 15 & -5.2 & -3.6 & 18.3 & 18.2 & 9.5 & 7.6 & 24.1 & 23.1 & 95.5 & 95.6 \\
\hline & 20 & -5.2 & -4.0 & 16.8 & 16.6 & 8.6 & 7.3 & 21.1 & 20.3 & 94.1 & 94.4 \\
\hline & 25 & 3.0 & 4.0 & 13.9 & 14.2 & -1.1 & -2.1 & 13.8 & 13.8 & 95.8 & 95.3 \\
\hline & 50 & -0.0 & 0.5 & 9.2 & 9.3 & 0.9 & 0.4 & 9.5 & 9.5 & 97.0 & 96.8 \\
\hline
\end{tabular}

Source: Own elaboration.

Sixth, the effect of the lack of Assumption A1 can be analyzed via the Figures 5 and 6, which are based on data extracted from the Gamma and Uniform distributions. The aim of Figures 5 and 6 is to estimate $C_{p k}=1$, with $k=0$, and we use $c=3$ for the control limits.

Finally, we use the pistonrings data to analyze the performance of different scenarios under this real situation (Figure 7). Obviously, we only have the case of a finite population. However, Assumption A2 may hold in this example if samples are selected with replacement. For this reason, Figure 7 contains samples selected with and without replacement. We considered $C_{p k}=1, k=0$, and $c=2.5$ for the simulation based on the pistonrings data. 
Figure 1

Values of Relative Bias (RB (\%)) and Relative Root Mean Square Error (RRMSE(\%)) for estimators of $\sigma=1(\mathrm{SD})$ and $\mathrm{C}_{\mathrm{p}}=\mathrm{C}_{\mathrm{pk}}=1(\mathrm{PCl})$, with $\mathrm{k}=0$, and empirical coverages (\%) of confidence intervals for $\mathrm{C}_{\mathrm{p}}=\mathrm{C}_{\mathrm{pk}}=1(\mathrm{PCl})$, with $\mathrm{k}=0$. Sample data are selected from a Normal distribution with $\mu=10$ and $\sigma=1$ (infinite population). For the finite population, $\mathrm{N}=500$ data are selected from this distribution and then samples without replacement are selected from this data set. Empirical measures are also obtained when the parameters $\mu$ and $\sigma$ are under control: statistics $\bar{x}$ and $S$ are within the corresponding control limits based on the constant $c=2.5$ (about $97 \%$ of the samples are in control). The $X$ axis shows the sample sizes $(n)$; and the sampling fractions $(f=n / N)$ in the case of finite populations

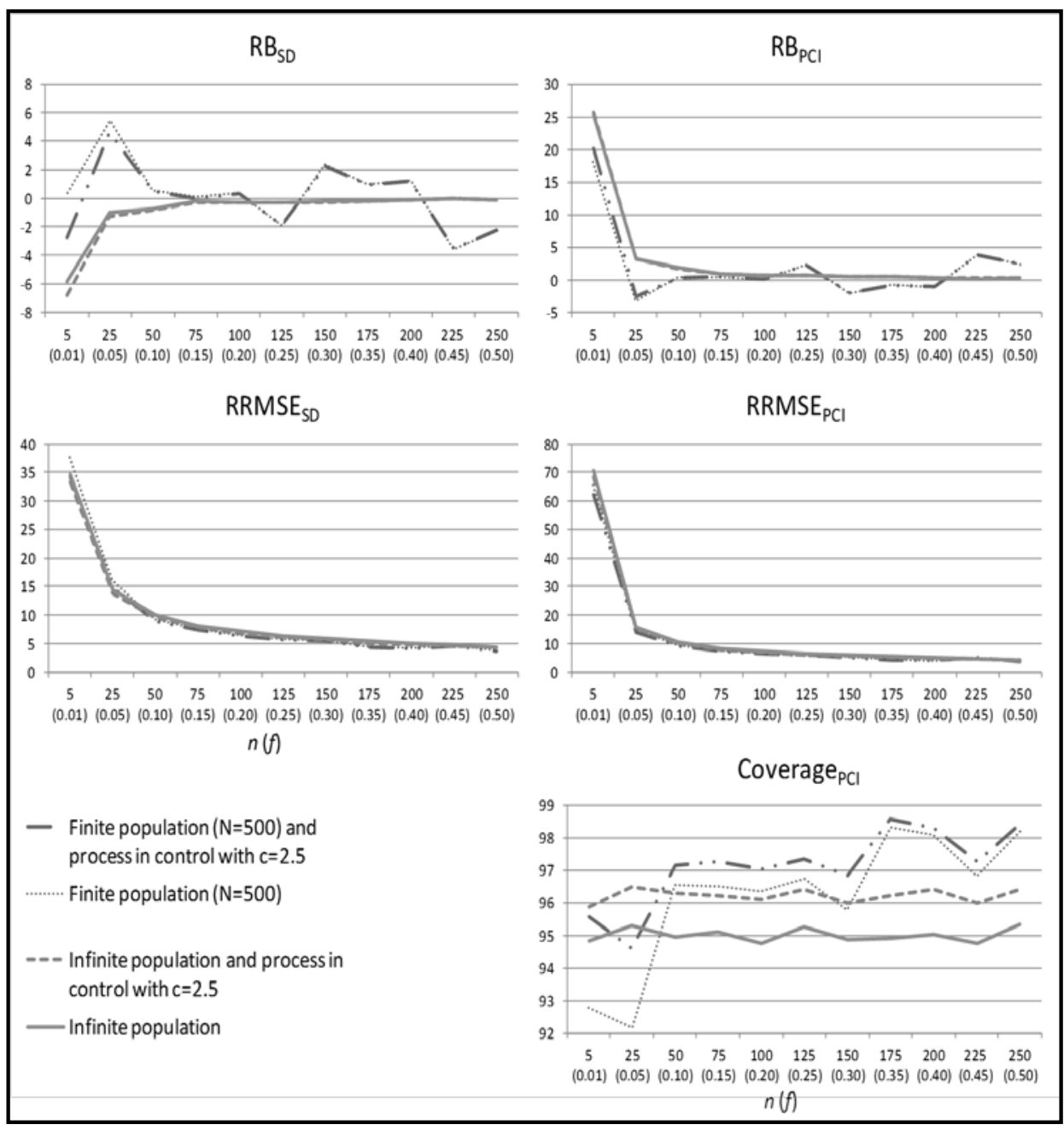

Source: Own elaboration. 
Figure 2

Values of Relative Bias (RB (\%)) and Relative Root Mean Square Error (RRMSE(\%)) for estimators of $\sigma=1(\mathrm{SD})$ and $\mathrm{C}_{\mathrm{pk}}=1(\mathrm{PCl})$, with $\mathrm{k}=0.25$, and empirical coverages (\%) of confidence intervals for $\mathrm{C}_{\mathrm{pk}}=1(\mathrm{PCl})$, with $\mathrm{k}=0.25$. Sample data are selected from a Normal distribution with $\mu=10$ and $\sigma=1$ (infinite population). For the finite population, $\mathrm{N}=500$ data are selected from this distribution and then samples without replacement

are selected from this data set. Empirical measures are also obtained when the parameters $\mu$ and $\sigma$ are under control: statistics $\bar{x}$ and $S$ are within the corresponding control limits based on the constant $c=2.5$ (about $97 \%$ of the samples are in control). The $X$ axis shows the sample sizes $(n)$; and the sampling fractions $(f=n / N)$ in the case of finite populations

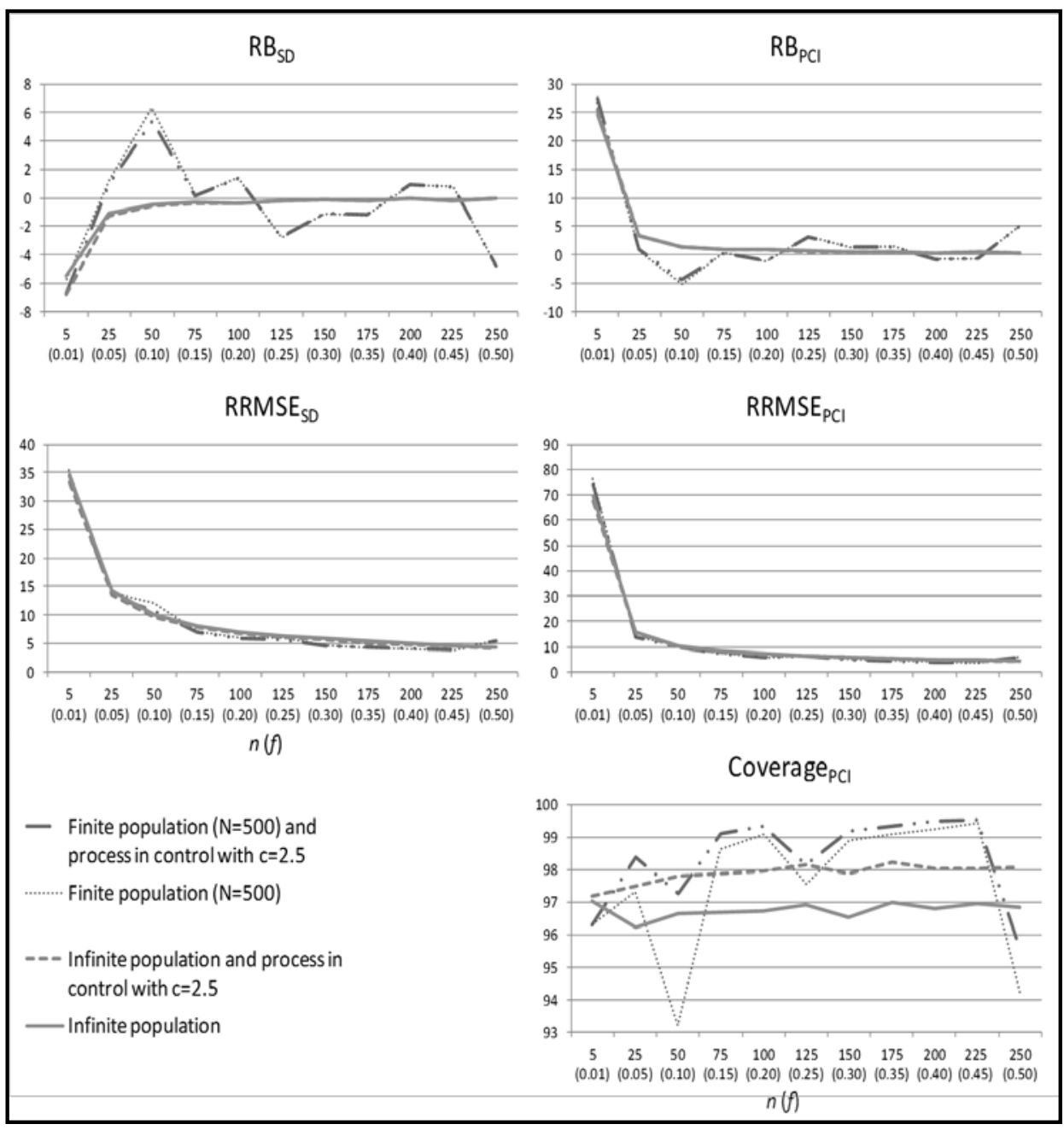

Source: Own elaboration. 
Figure 3

Values of Relative Bias (RB (\%)) and Relative Root Mean Square Error (RRMSE(\%)) for estimators of $\sigma=1(\mathrm{SD})$ and $\mathrm{C}_{\mathrm{pk}}=1(\mathrm{PCl})$, with $\mathrm{k}=0.5$, and empirical coverages (\%) of confidence intervals for $\mathrm{C}_{\mathrm{pk}}=1(\mathrm{PCl})$, with $\mathrm{k}=0.5$. Sample data are selected from a Normal distribution with $\mu=10$ and $\sigma=1$ (infinite population). For the finite population, $\mathrm{N}=500$ data are selected from this distribution and then samples without replacement

are selected from this data set. Empirical measures are also obtained when the parameters $\mu$ and $\sigma$ are under control: statistics $\overline{\mathrm{x}}$ and $\mathrm{S}$ are within the corresponding control limits based on the constant $c=2.5$ (about $97 \%$ of the samples are in control). The $X$ axis shows the sample sizes $(n)$; and the sampling fractions $(f=n / N)$ in the case of finite populations

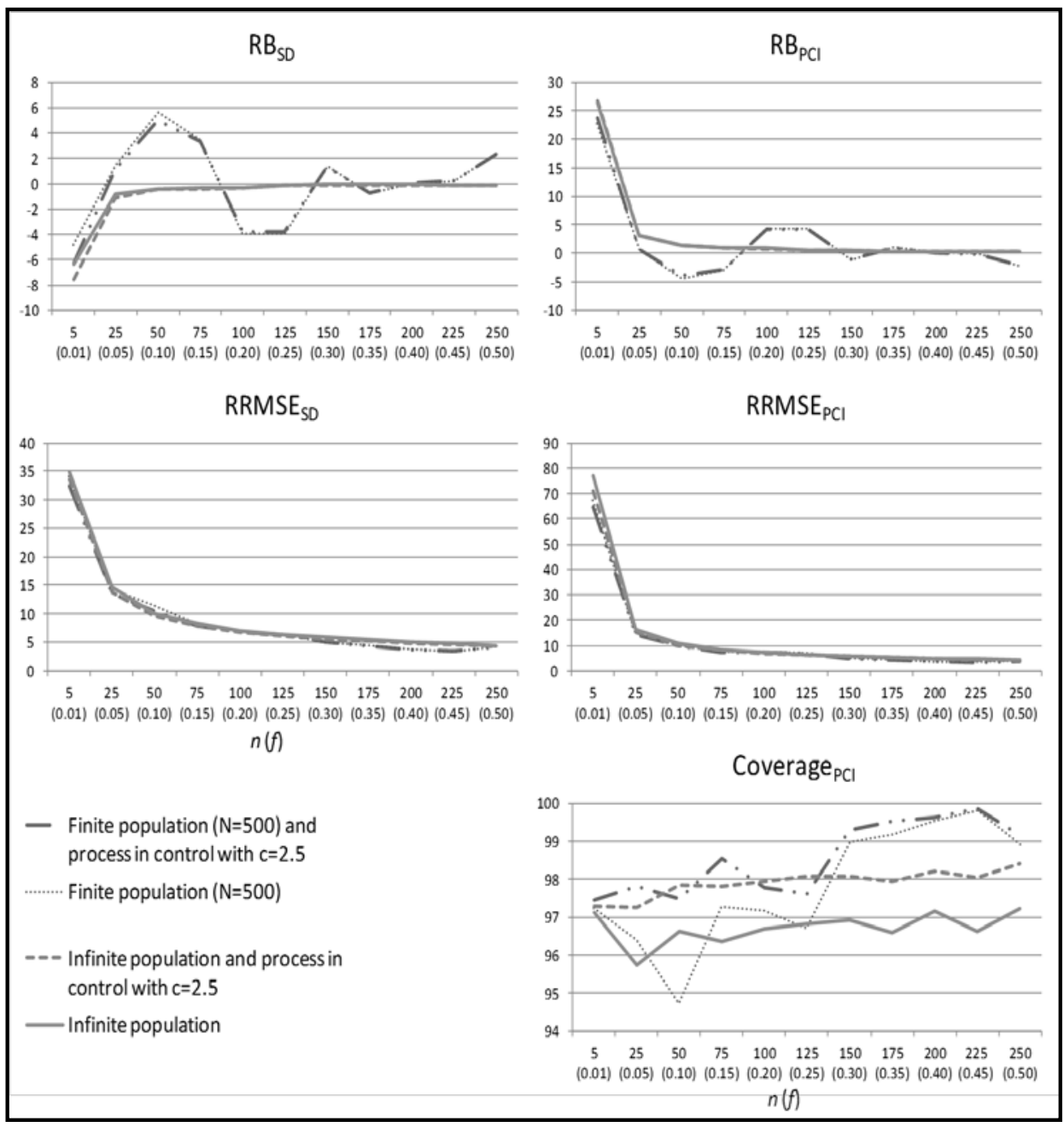

Source: Own elaboration. 
Figure 4

Values of Relative Bias (RB (\%)) and Relative Root Mean Square Error (RRMSE(\%)) for estimators of $\sigma=1(\mathrm{SD})$ and $\mathrm{C}_{\mathrm{p}}=\mathrm{C}_{\mathrm{pk}}=1(\mathrm{PCl})$, with $\mathrm{k}=0$, and empirical coverages (\%) of confidence intervals for $\mathrm{C}_{\mathrm{p}}=\mathrm{C}_{\mathrm{pk}}=1(\mathrm{PCl})$, with $\mathrm{k}=0$. Sample data are selected from a Normal distribution with $\mu=10$ and $\sigma=1$ (infinite population). For the finite population, $\mathrm{N}=10000$ data are selected from this distribution and then samples without replacement are selected from this data set. Empirical measures are also obtained when the parameters $\mu$ and $\sigma$ are under control: statistics $\bar{x}$ and $S$ are within the corresponding control limits based on the constant $c=2.5$ (about $97 \%$ of the samples are in control). The $X$ axis shows the sample sizes $(n)$; and the sampling fractions $(f=n / N)$ in the case of finite populations

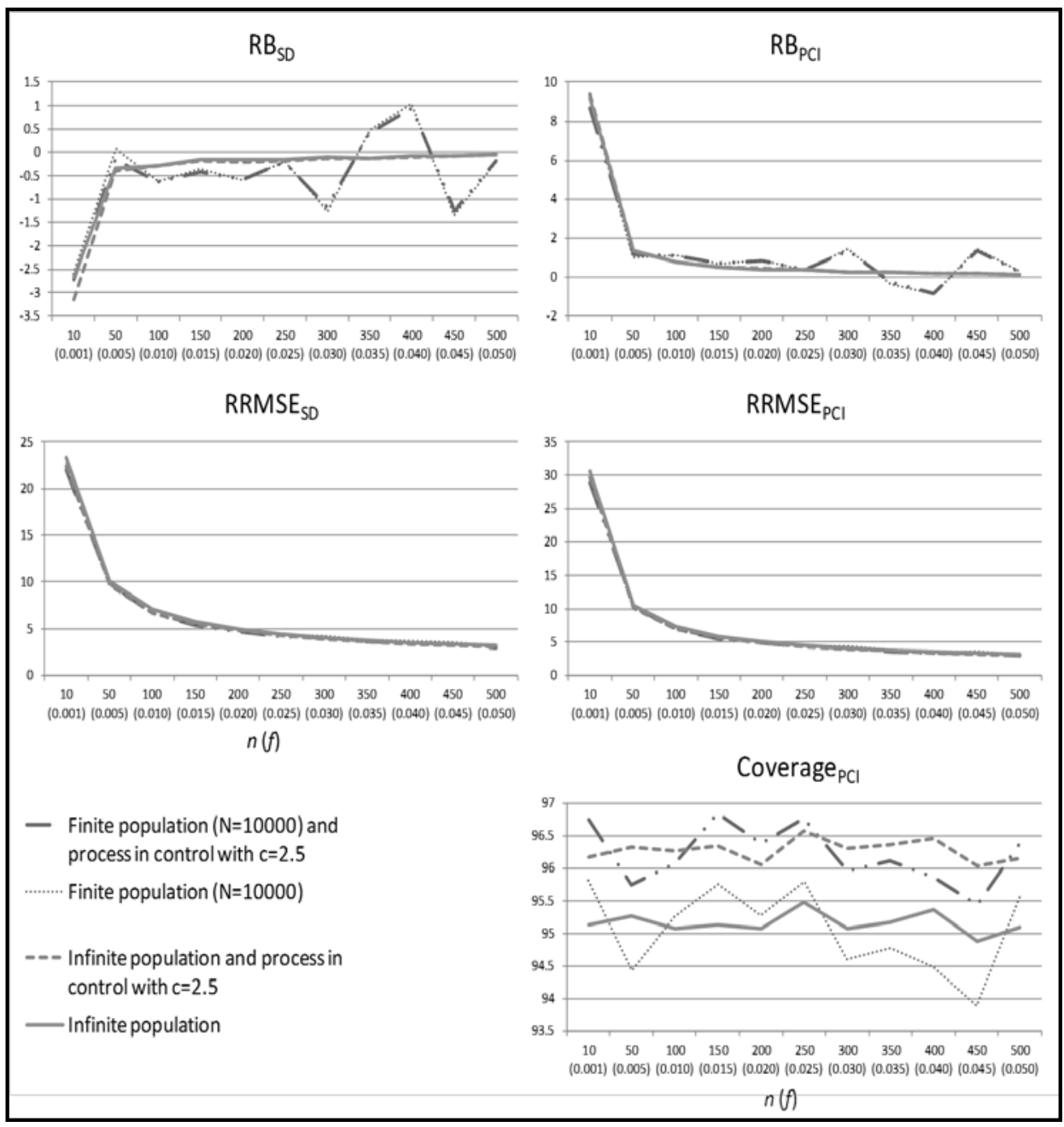

Source: Own elaboration. 
Figure 5

Values of Relative Bias (RB (\%)) and Relative Root Mean Square Error (RRMSE(\%)) for estimators of $\sigma=1(\mathrm{SD})$ and $\mathrm{C}_{\mathrm{p}}=\mathrm{C}_{\mathrm{pk}}=1(\mathrm{PCl})$, with $\mathrm{k}=0$, and empirical coverages (\%) of confidence intervals for $\mathrm{C}_{\mathrm{p}}=\mathrm{C}_{\mathrm{pk}}=1(\mathrm{PCl})$, with $\mathrm{k}=0$. Sample data are selected from a Gamma distribution with $\mu=10$ and $\sigma=1$ (infinite population). For the finite population, $\mathrm{N}=500$ data are selected from this distribution and then samples without replacement are selected from this data set. Empirical measures are also obtained when the parameters $\mu$ and $\sigma$ are under control: statistics $\bar{x}$ and $S$ are within the corresponding control limits based on the constant c $=3$ (about $99 \%$ of the samples are in control). The $X$ axis shows the sample sizes $(n)$; and the sampling fractions $(f=n / N)$ in the case of finite populations

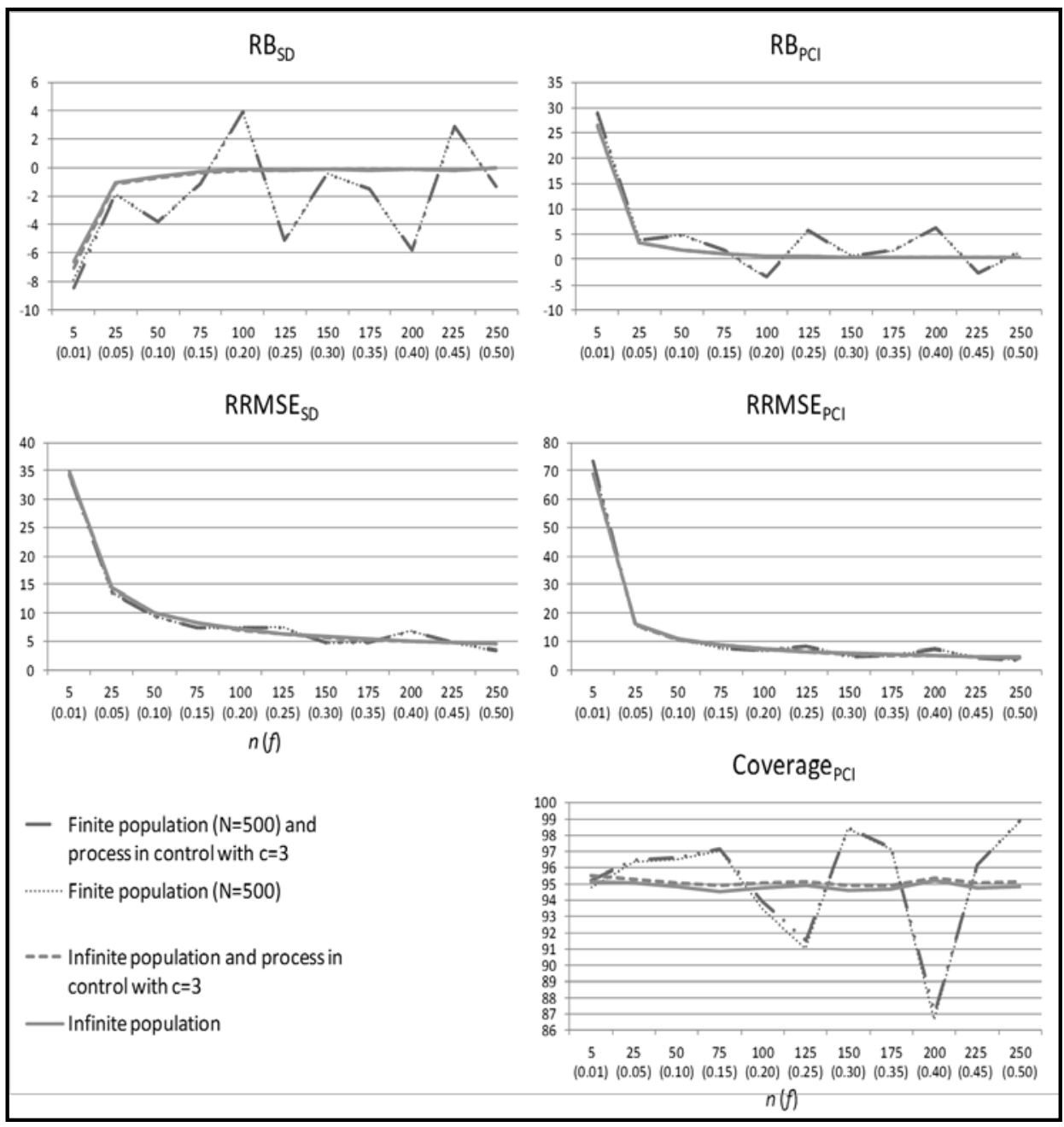

Source: Own elaboration. 
Figure 6

Values of Relative Bias (RB (\%)) and Relative Root Mean Square Error (RRMSE(\%)) for estimators of $\sigma=1(\mathrm{SD})$ and $\mathrm{C}_{\mathrm{p}}=\mathrm{C}_{\mathrm{pk}}=1(\mathrm{PCl})$, with $\mathrm{k}=0$, and empirical coverages (\%) of confidence intervals for $\mathrm{C}_{\mathrm{p}}=\mathrm{C}_{\mathrm{pk}}=1(\mathrm{PCl})$, with $\mathrm{k}=0$. Sample data are selected from a Uniform distribution with $\mu=10$ and $\sigma=1$ (infinite population). For the finite population, $\mathrm{N}=500$ data are selected from this distribution and then samples without replacement are selected from this data set. Empirical measures are also obtained when the parameters $\mu$ and $\sigma$ are under control: statistics $\bar{x}$ and S are within the corresponding control limits based on the constant c $=3$ (about $99 \%$ of the samples are in control). The $X$ axis shows the sample sizes $(n)$; and the sampling fractions $(f=n / N)$ in the case of finite populations

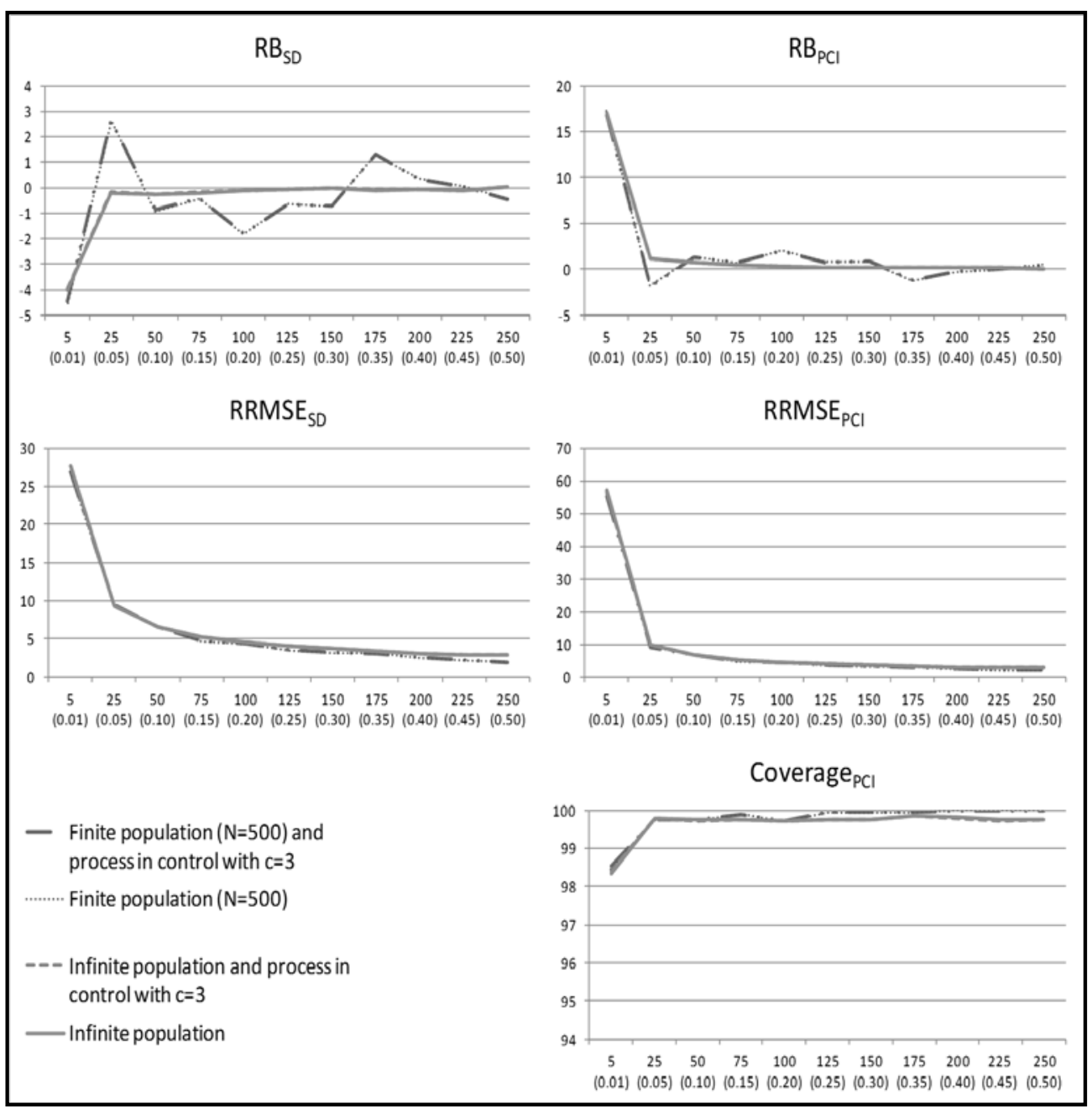

Source: Own elaboration. 
Figure 7

Values of Relative Bias (RB (\%)) and Relative Root Mean Square Error (RRMSE(\%)) for estimators of $\sigma=0.01291(\mathrm{SD})$ and $\mathrm{C}_{\mathrm{p}}=\mathrm{C}_{\mathrm{pk}}=1(\mathrm{PCl})$, with $\mathrm{k}=0$, and empirical coverages (\%) of confidence intervals for $\mathrm{C}_{\mathrm{p}}=\mathrm{C}_{\mathrm{pk}}=1(\mathrm{PCl})$, with $\mathrm{k}=0$. The pistonrings data set is the population considered, with $\mathrm{N}=125$. Samples are selected with and without replacement. Empirical measures are also obtained when the parameters $\mu$ and $\sigma$ are under control: statistics $\bar{x}$ and $S$ are within the corresponding control limits based on the constant $c=2.5$ (the percentage of samples in control ranges from $4 \%$ (when $f=0.05$ ) and $0.1 \%$ (when $f=0.5)$ ). The $X$ axis shows the sampling fractions $(f=n / N)$

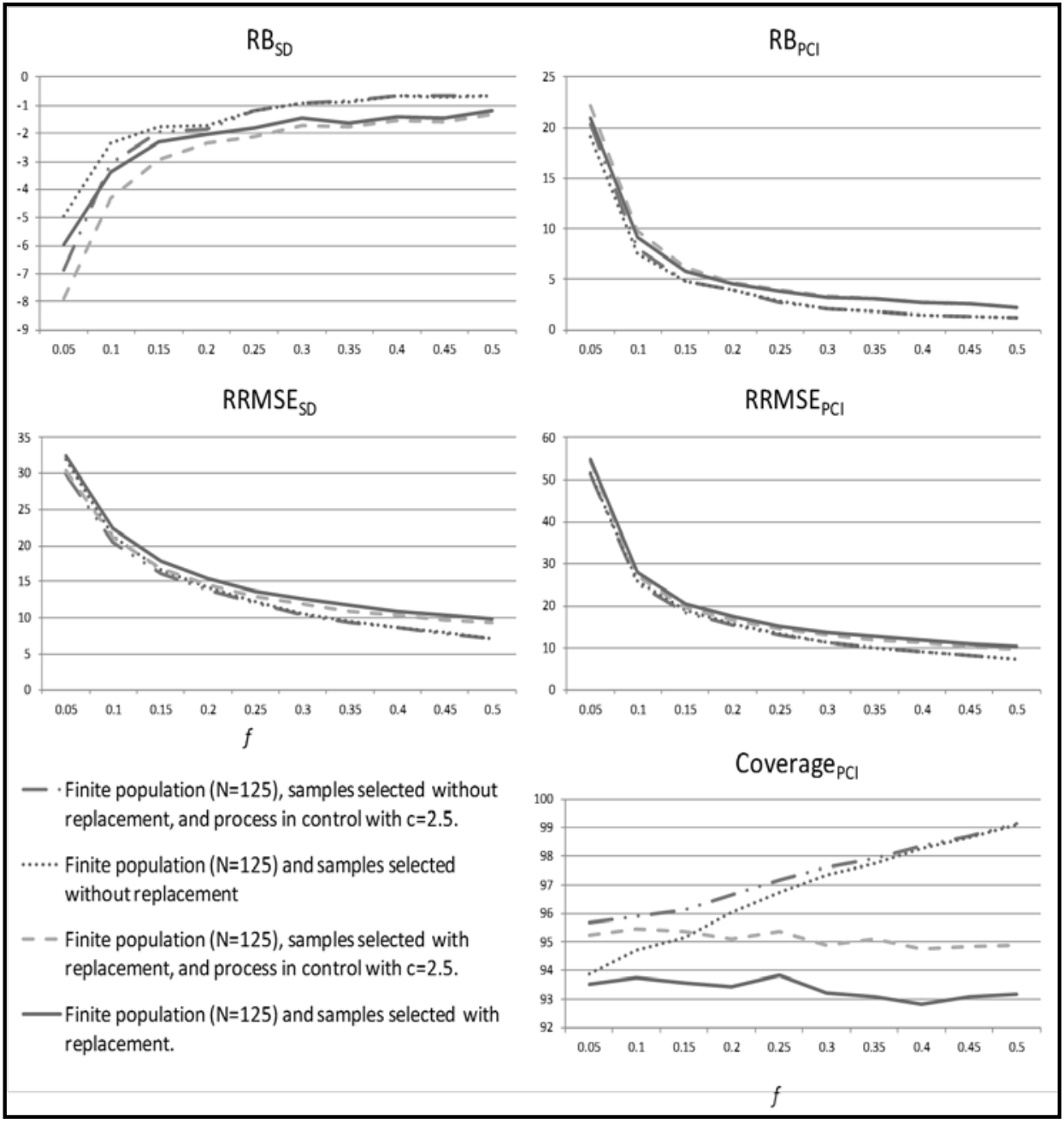

Source: Own elaboration. 


\subsection{Discussion of empirical results}

The performance of the estimators $\hat{\sigma}$ and $\hat{\sigma}_{c 4}$ can be compared in Table 1 . As we expected, $\hat{\sigma}$ has larger biases $\left(R B_{S D}\right)$ than $\hat{\sigma}_{c 4}$ when the sample size is small, and they perform similar as the sample size increases. $\hat{\sigma}_{c 4}$ can also have large biases in the case of finite populations and small sample sizes. If we analyze the accuracy of the estimators $\hat{\sigma}$ and $\hat{\sigma}_{c 4}$ in terms of $R R M S E_{S D}$, we can observe that both estimators have a similar performance, i.e., $\hat{\sigma}$ is slightly more accurate than $\hat{\sigma}_{c 4}$ under various sample sizes. The estimator of $C_{p}$ based on $\hat{\sigma}\left(\hat{C}_{p}\right)$ also has a larger bias than the estimator $\hat{C}_{p . c 4}$, and the most important differences are observed when the sample sizes are smaller than 20. $\hat{C}_{p . c 4}$ is generally more accurate than $\hat{C}_{p}$ in terms of $R R M S E_{P C I}$. Desirable empirical coverage rates of confidence intervals for $C_{p}$ are generally obtained, since such values are close to the required level of 95\%. Larger empirical coverage rates can be observed when $n=50$ and the population is finite.

Figure 1 gives the values of $R B_{S D}$ and $R R M S E_{S D}$ (in percentages) when the process standard deviation is $\sigma=1$ and the sample data are selected from a Normal distribution. Finite and infinite populations are considered. We observe that results derived from infinite populations give biases close to $0 \%$ when the sample size is larger than 25. The values of $R B_{S D}$ based on finite populations are also reasonable, but they generally range from $-4 \%$ to $2 \%$. A value of $R B_{S D}$ about $6 \%$ is obtained in this situation when $=25$. The various estimators have a similar performance in terms of $R R M S E_{S D}$. Figure 1 also gives the values of $R B_{P C I}$ and $R R M S E_{P C I}$ (in percentages) when estimating $C_{p}=1$. Conclusions derived from the relative biases are similar to the case of estimating $\sigma=1$, i.e., biases based on infinite populations are close to $0 \%$, and they range from $-5 \%$ to $5 \%$ in the case of finite populations (for sample sizes larger than 25). Large biases are observed when the sample sizes are smaller than 25. Results derived from Figure 1 can be also used to analyze the Assumption A3, i.e., the fact that the process is in control when estimators and confidence intervals are obtained. We observe that relevant differences are not observed in terms of bias $(R B)$ and accuracy (RRMSE) of estimators. However, we observe that confidence intervals obtained when the process is in control, and using the constant $c=2.5$ for the control limits, have a larger coverage rate than the case where the Assumption A3 is not completely satisfied. Note that this difference appears when $c=2.5$, whereas similar coverage rates are obtained under the standard situation (when $c=3$ ).

The process is centered in Figure 1. Off-center processes are considered in Figures 2 and 3, where $C_{p k}=1$, with $k=\{0.25,0.5\}$, respectively. We observe that the value of $k$ does not have a relevant impact on the bias and accuracy of estimators, since the values of $R B$ and RRMSE of Figures 1, 2 and 3 are similar. 
However, we observe that the value of $k$ may have an impact on the coverage rates, since such values are larger than $95 \%$ when $k=\{0.25,0.5\}$.

The population size is not large in Figure $1(N=500)$. Figure 4 has the same conditions than Figure 1, except on the fact that the population size is $N=10000$ in Figure 4. This implies that the population is finite, but the approximation to an infinite population may fit well. The quality of this approximation can be also observed if we compare the results derived from finite and infinite populations in Figure 4, i.e., they provide similar coverage rates in this situation. However, we obtain different coverage rates in Figure 1.

If we analyze the lack of the normality (Assumption A1) using the Gamma distribution and infinite populations (Figure 5), we observe that the impact is not important in comparison to results derived under normality (see, for example, Figure 1). Assuming finite populations, the coverage rates can have more variability in the scenario of the Gamma distribution (see also Figure 5). If we analyze a more extreme situation (the Uniform distribution; Figure 6), the most relevant impact can be observed in the confidence intervals, since the corresponding coverage rates are close to $100 \%$. This is the situation for both finite and infinite populations.

Similar conclusions are obtained under the real data set. First, the biases are closer to $0 \%$ as the sampling fraction increases. The different scenarios give similar values of RB and RRMSE. As far as the coverage rates is concerned, we observe a good performance when the Assumption A2 holds (samples selected with replacement). However, coverage rates have a worse performance as the sampling fraction increases, and assuming the scenario of samples selected without replacement.

\section{CONCLUSIONS AND FUTURE RESEARCH}

The most important conclusions derived from this paper are summarized in this section. Some future research lines are also introduced.

First, we analyze two different estimators for the standard deviation and the capability index. As we expected, the biased estimator has larger biases when the sample sizes are smaller than 10, and they perform similar otherwise. They have a similar performance in terms of accuracy and empirical coverage.

In addition, we also analyze center and off-center processes, i.e., different values of $k$ are considered. Results indicate that the value of $k$ does not have a relevant impact on the bias and accuracy of estimators, but values of $k$ larger than 0 may produce confidence intervals with larger coverage rates.

Estimators and confidence intervals for the capability index are based on the Assumptions A1, A2, A3, which are described in Section 2.2. Assumption A1 indicates that the quality characteristic follows a Normal distribution. Desirable 
coverage rates are obtained if we consider the Gamma distribution and infinite populations. In the extreme case of a Uniform distribution, coverage rates close to $100 \%$ are obtained, under both finite and infinite populations.

Assumption A2 establishes that the variables associated to the sample values are independent and identically distributed. We have assumed this assumption by considering sample data extracted from infinite populations, and it was not satisfied when sample data were selected from finite populations. The first effect of the lack of this assumption is the presence of biases with a greater variation, but they also give reasonable values. This assumption does not have a relevant impact on the accuracy, i.e., on the values of RRMSE. The second effect can be observed in the coverage rates. Confidence interval for the capability index and based on finite population can have coverage rates larger than the required nominal level. The commented effects are not observed under finite populations with a large population size.

Assumption A3 indicates that the process capability index may be applied when the process is in control (see Montgomery 2009, p.181, Evans and Lindsay 1999, p.321, Jensen et al., 2006). Two different situations are analyzed in this paper: (i) we only used samples whose statistics are within the control limits (the process is in control); and (ii) we considered all samples without checking if the process is in control. Note that the fact of using random values selected from probabilistic distribution provides a large percentage of samples in control, which is the common situation in practice. Similar results are obtained in terms of bias and accuracy. However, both situations may give different coverage rates for the confidence intervals of the process capability index.

Conclusions derived from this paper suggest some future researches on this topic. For instance, it could be interesting to analyze the different scenarios of this paper for estimators and confidence intervals based on the sample range, instead of the sample standard deviation used in this paper. For this purpose, the construction of confidence intervals with desirable properties and based on the sample range is required. In addition, efforts could be made to improve coverage rates of confidence intervals for the process capability index in the case of finite populations. For instance, confidence intervals based on techniques such as linearization, resampling methods (bootstrap or jackknife) or the balanced repeated replication (Wolter 2007) could be analyzed for the suggested scenarios. 


\section{REFERENCES}

ÁLVAREZ, E.; MOYA-FÉRNANDEZ, P.J.; BLANCO-ENCOMIENDA, F.J. and MUÑOZ, J.F. (2015). "Methodological insights for industrial quality control management: The impact of various estimators of the standard deviation on the process capability index". Journal of King Saud University-Science, 27, pp. 271-277.

ASLAM, M.; AZAM, M.; WU, C. and JUN, C. (2015). "Mixed acceptance sampling plans for product inspection using process capability index". Quality Control and Applied Statistics, 60, pp. 455-486.

BESTERFIELD, D (2014).: Quality improvement ( $9^{\text {th }}$ edn). Harlow: Pearson Education.

BREYFOGLE, F.W. (2003). Implementing six sigma - smarter solutions using statistical methods. $2^{\text {nd }}$ Edition. New Jersey: John Wiley \& Sons, Inc.

SCRUCCA, L. (2004). "qcc: an R package for quality control charting and statistical process control". R News 4 (1). pp. 11-17.

CHAKRABORTI, S.; HUMAN, S.W. and GRAHAN, M.A. (2008). "Phase I Statistical Process Control Charts: An overview and some results". Quality Engineering, 21, pp. 52-62.

CHEN, G. (1997). "The mean and standard deviation of the run length distribution of Xbar charts when control limits are estimated". Statistica Sinica, 7, pp. 789-798.

CHOU, Y.M. and OWEN, D.B. (1989). "On the distribution of the estimated process capability indices". Communications in Statistics - Theory and Methods, 18, pp. 45494560.

CHOU, Y.M.; OWEN, D.B. and BORREGO, A.S.A. (1990). "Lower confidence limits on process capability indices". Journal of Quality Technology, 22, pp. 223-229.

DOVICH, R. A. (1992). Quality engineering statistics, Milwaukee,Wisconsin: ASQ Quality Press.

DUNCAN, A.J. (1986). Quality control and industrial statistics, $5^{\text {th }}$ ed. Homewood, IL: Richard D. Irwin.

EVANS, J. R. and LINDSAY, W. M. (1999). The management and control of quality. $8^{\text {th }}$ ed. South Western College.

FRANKLIN, L.A. and WASSERMAN, G.S. (1992) "A note on the conservative nature of the tables of lower confidence limits for $\mathrm{C}_{\mathrm{pk}}$ with a suggested correction". StatisticsSimulation and Computation, 21(4), pp. 1165-1169.

GUIRGUIS, G. H. and RODRIGUEZ, R.N. (1992). "Computation of Owen's Q function applied to process capability analysis". Journal of Quality Technology, 24(4), pp. 236246.

HARMS, T. and DUCHESNE, P. (2006). "On calibration estimation for quantiles". Survey Methodology, 32, pp.37-52.

HEAVLIN, W. D. (1988). "Statistical properties of capability indices". Technical report, 320, Tech. Library, Advanced Micro Devices, Inc., Sunnyvale, California.

JENSEN, W. A.; JONES-FARMER, L. A.; CHAMP, C. W. and Woodall, W. H. (2006). "Effects of parameter estimation on control chart properties: a literature review". Journal of Quality Technology, 38(4), pp. 349-364. 
JONES, L.A.; CHAMP, C.W. and RIGDON, S.E. (2001). "The performance of exponentially weighted moving average charts with estimated parameters". Technometrics, 43(2), pp. 156-167.

KANE, V.E. (1986). "Process capability indices". Journal of Quality Technology, 18(1), pp. 41-52.

KOTZ, S. and JHONSON, N.L. (1993). Process capability indices. New York: CRC Press.

KOTZ, S. and JHONSON, N.L. (2002). "Process capability indices: a review 1992-2000. Discussions". Journal of Quality Technology, 34(1), pp. 2-19.

KOTZ, S. and LOVELACE, C.R. (1998). Process capability indices in theory and practice. London: Arnold.

KUSHLER, R.H. and HURLEY, P. (1992). "Confidence bounds for capability indices". Journal of Quality Technology, 24(4), pp. 188-195.

LI, H.; OWEN, D.B. and BORREGO, A.S.A. (1990). "Lower confidence limits on process capability indices based on the range". Communications in Statistics - Simulation and Computation, 19, pp. 1-24.

LUKO, S.N. (1996). "Concerning the estimators $\bar{R} / d_{2}$ and $\bar{R} / d_{2}^{*}$ in estimating variability in a Normal Universe". Quality Engineering, 8 (3), pp. 481-487.

MITRA, A. (2008). Fundamentals of quality control and improvement. New York: Wiley.

MONTGOMERY, D.C. (1985). Introduction to statistical quality control. New York: Wiley.

MONTGOMERY, D.C. (2009). Statistical quality control. A modern introduction (6 $6^{\text {th }}$ edn). New York: Wiley.

MUÑOZ, J.F.; ÁLVAREZ-VERDEJO, E.; PÉREZ-ARÓSTEGUI, M.N. and GUTIÉRREZGUTIÉRREZ, L. (2016). "Empirical comparisons of X-bar charts when control limits are estimated". Quality and Reliability Engineering International, 32, pp. 453-464.

NAGATA, Y. and NAGAHATA, H. (1994). "Approximation formulas for the lower confidence limits of process capability indices". Okayama Economic Review, 25(4), pp. 301-314.

NEZHAD, M. and NIAKI, S. (2013). "A new acceptance sampling policy based on number of successive conforming items". Communications in Statistics - Theory and Methods, 42, pp. 1542-1552.

OTT, E.R. (1975). Process quality control. New York: McGraw-Hill.

RAO, J. N. K.; KOVAR, J. G. and MANTEL, H. J. (1990). "On estimating distribution functions and quantiles from survey data using auxiliary information". Biometrika, 77, pp. 365-375.

RAO, G.S.; ROSAIAH, K.; BABU, M. and SIVAKUMAR, C. (2016). "New acceptance sampling plans based on percentiles for exponentiated Fréchet distribution". Economic Quality Control, DOI: 10.1515/eqc-2015-0011.

SILVA, P. N. and SKINNER, C. J. (1995). "Estimating distribution functions with auxiliary information using poststratification". Journal of Official Statistics, 11(5), pp. 277-294.

SPIRING, F.; LEUNG, B.; CHENG, S. and YEUNG, A. (2003). "A bibliography of process capability papers". Quality and Reliability Engineering International, 19(5), pp. 445460.

VARDEMAN, S.B. (1999). "A brief tutorial on the estimation of the process standard deviation". IIE Transact, 31, pp. 503-507. 
WHEELER, D.J. (1995). Advanced topics in statistical process control. Knoxville, TN: SPC press.

WOLTER, K.M. (2007). Introduction to variance estimation. $2^{\text {nd }}$ ed. New York: Springer.

WOODALL, W.H. and MONTGOMERY, D.C (2000) "Using ranges to estimate variability". IIE Quality Engineering, 13(2), pp. 211-217. 